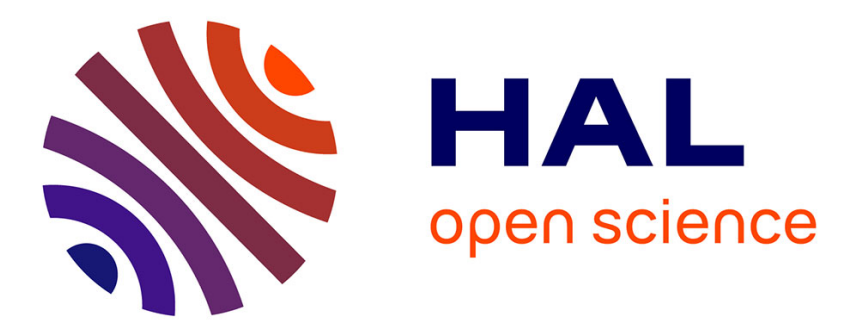

\title{
Multimode Brillouin spectrum in a long tapered birefringent photonic crystal fiber
}

Joël Cabrel Tchahame, Jean-Charles Beugnot, Alexandre Kudlinski, Thibaut

Sylvestre

\section{- To cite this version:}

Joël Cabrel Tchahame, Jean-Charles Beugnot, Alexandre Kudlinski, Thibaut Sylvestre. Multimode Brillouin spectrum in a long tapered birefringent photonic crystal fiber. Optics Letters, 2015, 40 (18), pp.4281-4284. 10.1364/OL.40.004281 . hal-01198791

\section{HAL Id: hal-01198791 \\ https://hal.science/hal-01198791}

Submitted on 14 Sep 2015

HAL is a multi-disciplinary open access archive for the deposit and dissemination of scientific research documents, whether they are published or not. The documents may come from teaching and research institutions in France or abroad, or from public or private research centers.
L'archive ouverte pluridisciplinaire HAL, est destinée au dépôt et à la diffusion de documents scientifiques de niveau recherche, publiés ou non, émanant des établissements d'enseignement et de recherche français ou étrangers, des laboratoires publics ou privés. 


\title{
Multimode Brillouin spectrum in a long tapered birefringent photonic crystal fiber
}

\author{
Joël Cabrel Tchahame ${ }^{1}$, Jean-Charles Beugnot ${ }^{1}$, Alexandre Kudlinski ${ }^{2}$, and Thibaut Sylvestre ${ }^{1, *}$ \\ ${ }^{1}$ Institut FEMTO-ST, CNRS UMR 6174, Université de Franche-Comté, Besançon, France \\ ${ }^{2}$ Laboratoire PhLAM, I RCICA, UMR 8523 CNRS, Université de Lille 1, 59655 Villeneuve D'Ascq Cedex \\ *Corresponding author: thibaut.sylvestre@univ-fcomte.fr
}

\author{
Compiled August 19, 2015
}

\begin{abstract}
We investigate the stimulated Brillouin scattering (SBS) in a long tapered birefringent solid-core photonic crystal fiber (PCF) and compare our results with a similar but untapered PCF. It is shown that the taper generates a broadband and multi-peaked Brillouin spectrum, while significantly increasing the threshold power. Furthermore, we observe that the strong fiber birefringence gives rise to a frequency shift of the Brillouin spectrum which increases along the fiber. Numerical simulations are also presented to account for the taper effect and on the birefringence. Our findings open a new mean to control or inhibit the SBS by tapering photonic crystal fibers. (c) 2015 Optical Society of America
\end{abstract}

OCIS codes: $060.0060,290.5900,060.5295,190.4370,320.7140$

Stimulated Brillouin scattering (SBS) in optical fibers is a nonlinear optical effect that results from the interaction between light and acoustic waves [1]. It has important implications in optical fibers since it is ultimately responsible for restricting the light power that can be transmitted [1]. An increasingly important number of applications to telecommunications and optical sensors however, rely on SBS, which can become widely tailorable and highly sensitive to temperature and strain $[1,2,3,4]$. SBS thus plays a central role for the development of nonlinear optical technologies and continues to be area of active research both from a fundamental and applied viewpoints. In this context, SBS has recently been the subject of a renewed interest in sub-wavelength optical waveguides such as small-core photonic crystal fibers (PCFs), tapered optical fibers, nanoscale silicon or chalcogenide chip waveguides $[5,6,7,8,9,10,11]$. Significantly, although it was widely considered that SBS in standard optical fibers involves a single bulk longitudinal acoustic wave, SBS in highly confined waveguides can result from multiple hybrid shear and longitudinal, and even surface acoustic waves [5,8]. It has also been demonstrated that boundary radiation pressure in nanoscale waveguides can strongly enhance the SBS gain $[9,10]$.

In this Letter, we show that new Brillouin dynamics also occurs in a long adiabatically-tapered silica PCF where the core size shrinks from $2 \mu \mathrm{m}$ down to $800 \mathrm{~nm}$. Our measurements show in particular the generation of a broadband and multi-peaked Brillouin spectrum in comparison with an untapered PCF, while increasing the SBS threshold. Furthermore, we observe that the birefringence induces a large frequency shift of the whole SBS spectrum in both fibers. Numerical simulations including the variations of core size are also presented.

Figure 1(a) shows the longitudinal evolution of the core-decreasing PCF measured during the drawing process. As can be seen, its outer diameter decreases linearly from 205 to $80 \mu \mathrm{m}$ over $124 \mathrm{~m}$. The insets show a scan-
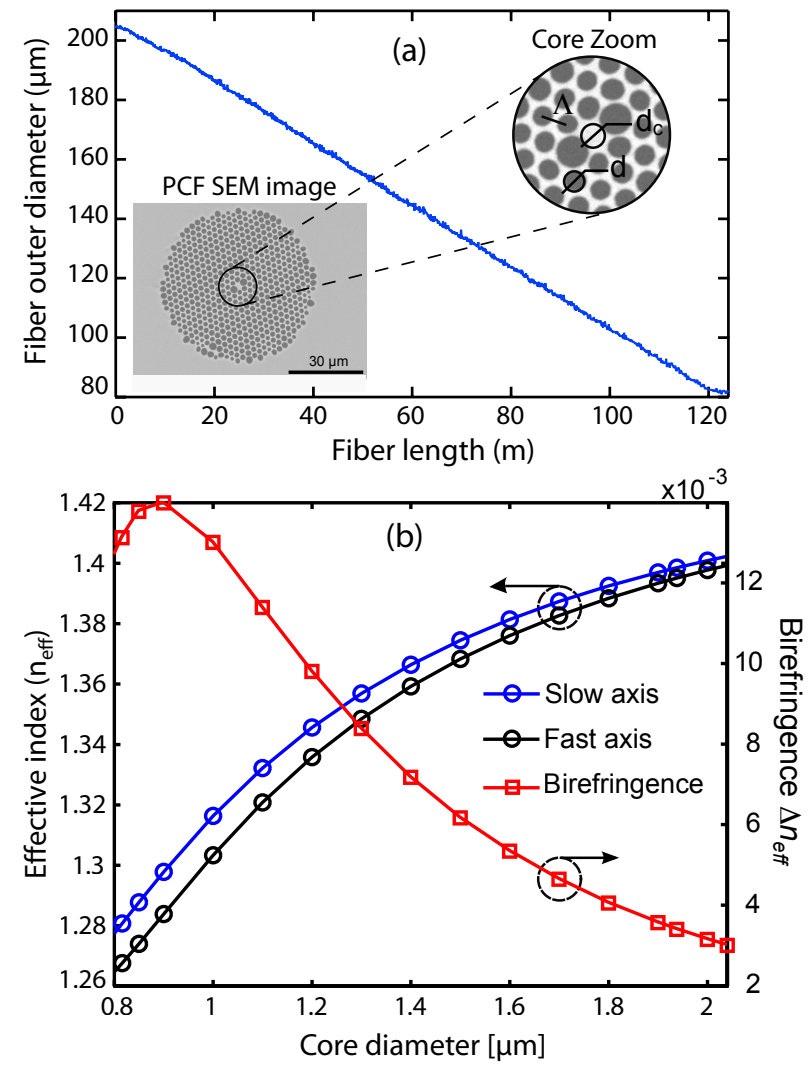

Fig. 1. (a) Fiber outer diameter versus length measured during drawing. Insets: SEM image of fiber input cross section with a zoom to center: $\mathrm{d}_{c}=2 \mu \mathrm{m} ; \mathrm{d}=1.9 \mu \mathrm{m} ; \Lambda=2.5 \mu \mathrm{m}$; linear attenuation at $1.55 \mu \mathrm{m}$ is $0.104 \mathrm{~dB} . \mathrm{m}^{-1}$. (b) Computed effective refractive indices and birefringence at $1.55 \mu \mathrm{m}$ versus the core diameter variation along the fiber.

ning electron microscope (SEM) image of the input PCF cross-section and a zoom to the core region. The output structure was preserved in a homothetic way with a ra- 


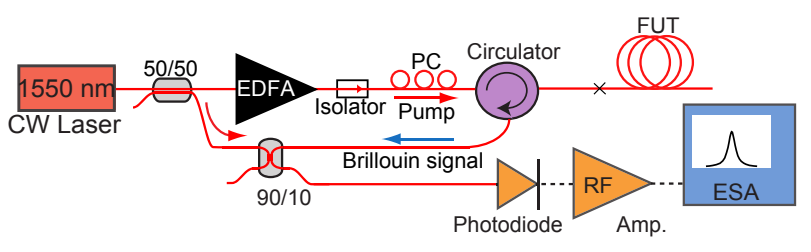

Fig. 2. Experimental setup used to measure the Brillouin spectrum. EDFA: Erbium Doped Fiber Amplifier; PC: polarization controller; FUT: fiber under test; CW: continuous wave; ESA: Electrical Spectrum Analyzer; Amp.: RF amplifier.

tio $H=\mathrm{d}_{\text {out }} / \mathrm{d}_{\text {in }}$ of about 0.4 . Two bigger holes were inserted around the core to make it elliptical, therefore enhancing its form-induced birefringence. The input core size is about $2 \times 3 \mu \mathrm{m}$ and decreases down to $0.8 \times 1.17 \mu \mathrm{m}$ over the $124 \mathrm{~m}$-long fiber. In the following, we note the core diameter $d_{c}$ which corresponds to the shortest axis of the elliptical core (see inset of Fig. 1(a)). From the microstructure cross section, we then computed the effective refractive index of the fundamental mode at 1.55 $\mu \mathrm{m}$ as a function of the core diameter. The results are shown in Fig. 1(b) for the slow and fast axis of the birefringent fiber. The birefringence is also plotted in red. It varies significantly from $3.10^{-3}$ to $1.4 .10^{-2}$ along the tapered fiber. From the acoustic point of view, the longitudinal variations of effective indices theoretically would yield BFS of almost $1 \mathrm{GHz}$, between 9.81 and $10.75 \mathrm{GHz}$. This range can be readily calculated from the standard formula $\nu_{\mathrm{B}}=2 n_{\mathrm{eff}} V_{\mathrm{L}} / \lambda$, where $\nu_{\mathrm{B}}$ is the Brillouin frequency shift; $n_{\text {eff }}$ is the effective index; $V_{\mathrm{L}}=5960 \mathrm{~m} . \mathrm{s}^{-1}$ is the longitudinal acoustic wave velocity in silica [1], and $\lambda=1.55 \mu \mathrm{m}$ is the optical wavelength.

Figure 2 depicts the experimental setup used for Brillouin measurements [6]. The pump laser is a linearlypolarized narrow-linewidth continuous-wave laser running at a wavelength of $1550 \mathrm{~nm}$. It was split into two beams by using a 50:50 fiber tap coupler (FC). One part of the beam was amplified by an Erbium doped fiber amplifier (EDFA) and injected in the tapered PCF through an optical circulator. The PCF was spliced to a standard single-mode fiber pigtail using an intermediate high-numerical aperture (HNA) fiber to optimize the coupling efficiency. Total insertion loss was $2.5 \mathrm{~dB}$. We also incorporated in the setup a polarization controller, so as to observe the role of birefringence on the Brillouin spectrum and threshold. The Brillouin signal coming back from the PCF was coupled to the pump beam using a 90:10 coupler. The resulting beat signal was detected by a fast photodiode and average Brillouin spectra were recorded with an electrical spectrum analyzer (ESA) in the RF domain around $10.5 \mathrm{GHz}$ with a point every $200 \mathrm{kHz}$. We compared our tapered PCF with a similar but untapered PCF with a length of $47 \mathrm{~m}$ and a constant core size $\mathrm{d}_{c}=2 \mu \mathrm{m}$.

Figure 3(a) and (b) show the experimental Brillouin spectra for the two tapered and untapered PCFs and
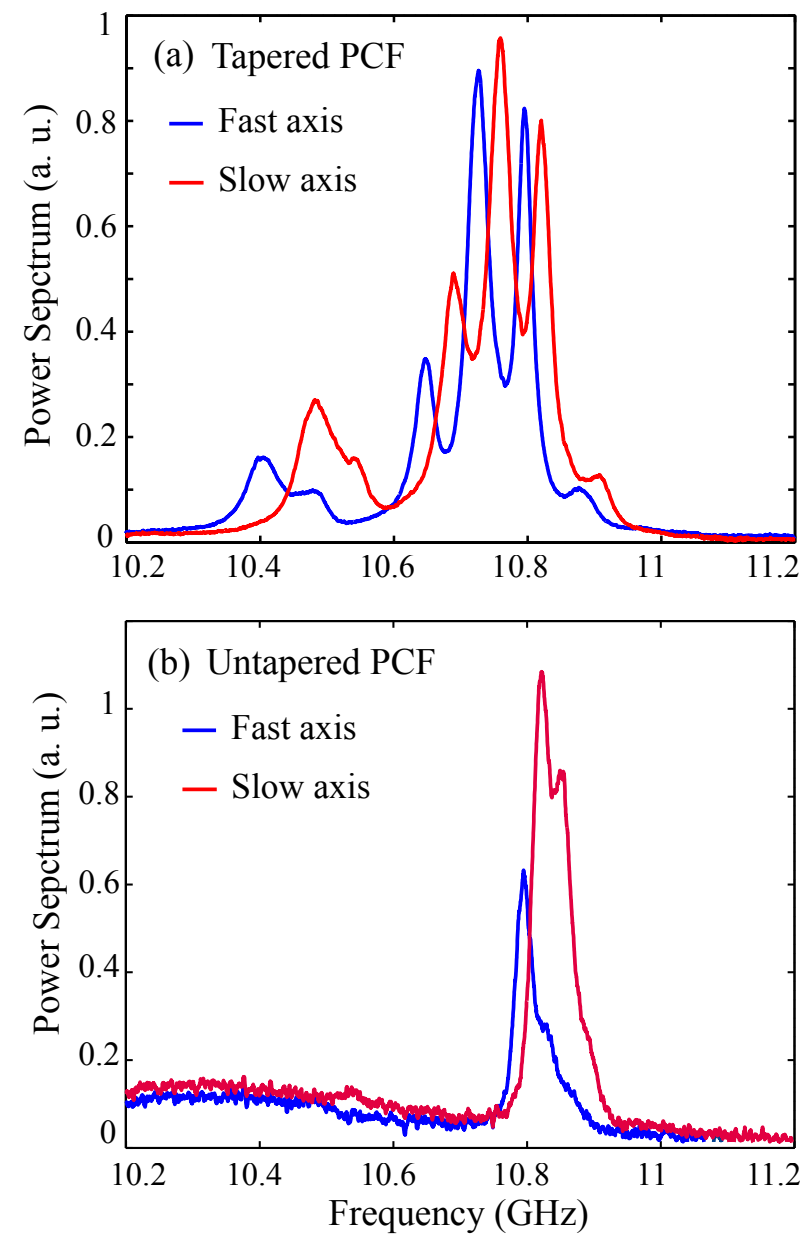

Fig. 3. Experimental Brillouin spectra measured for two input states of polarization in (a) the tapered PCF and (b) the untapered PCF. The pump power is $24.7 \mathrm{dBm}$ and 22.3 $\mathrm{dBm}$, respectively. The birefringence-induced frequency shift between the two main peaks is $33 \mathrm{MHz}$ and $26 \mathrm{MHz}$, respectively. Resolution is $200 \mathrm{KHz}$.

for two input states of polarization. As can be seen, the top figure exhibits a broad Brillouin spectrum spanning almost $500 \mathrm{MHz}$ and including several resonance peaks. Specifically, we can find in both polarizations 5 frequency-detuned acoustic peaks with different weight and linewidth. A further comparison with the Brillouin spectra of the untapered clearly shows the role of the taper to decreases the BFS and broadens the spectrum along the fiber. Intuitively, one would expect the probability function to be rectangular, and if so, the spectrum of the tapered PCF should be much more smeared out, instead of containing several peaks. We believe that this is actually due to the generation of several hybrid acoustic waves at different position along the fiber with a strong gain competition among them. In small-core PCFs, light strongly interacts with the airhole microstructure and the waveguide boundaries, leading to the generation of several hybrid elastic modes with dierent BFS $[5,8]$. Correspondingly, this effect strongly ehance the threshold power. For comparison, the Bril- 
louin spectrum of untapered PCF has almost a single peak at $10.8 \mathrm{GHz}$ with a linewidth of $50 \mathrm{MHz}$ (FWHM). We can see however a secondary peak in the red spectrum, which could be attributed to the fact that some acoustic modes can rotate the polarization state whereas other do not [12]. In addition, the red and blue Brillouin spectra in Figs 3(a) and (b) also highlights the influence of fiber birefringence. This results in a large frequency shift due the refractive index change between the two orthogonal polarizations, as shown in Fig. 1(b). Interestingly, for the tapered PCF, the birefringence-induced frequency shift between the multiple peaks significantly increases when the BFS decreases. This behavior confirms that the birefringence increases with the tapered fiber length. From the two main peaks we estimated a frequency shift of about $33 \mathrm{MHz}$ between the two spectra, which would correspond to an average birefringence of $4.3 .10^{-3}$, and thus a core size of $1.7 \mu \mathrm{m}$. For the untapered PCF, we measured a BFS shift of $26 \mathrm{MHz}$ between the two states of polarization. Theoretically, the birefringence of this untapered PCF is $3 \times 10^{-3}$ and this would yield to a Brillouin frequency shift of $\sim 23 \mathrm{MHz}$, which is in quite good agreement with the experiment [13]. The measurements also shows the impact of birefringence on Brillouin backscattering in terms of efficiency and threshold [14].

To get better insight, we performed numerical simulations based on the elastodynamics equation that governs the light-sound interaction in optical fibers [15]. This equation reads as

$$
\rho \frac{\partial^{2} u_{i}}{\partial t^{2}}-\left(c_{i j k l} u_{k, l}\right)_{, j}=-T_{i j, j}^{e s}
$$

Where $\rho$ is the material density, $u_{i}$ the displacement, $c_{i j k l}$ the rank-4 tensor of elastic constants. $T_{i j}^{e s}=$ $-\epsilon_{0} \chi_{k l i j} E_{k} E_{l}^{*}$ is the electrostrictive stress tensor, with the rank-4 susceptibility tensor $\chi_{k l i j}=\varepsilon_{k m} \varepsilon_{l n} p_{m n i j}$ and $p_{m n i j}$ the elasto-optic tensor, $\epsilon_{0}$ is the permittivity of vacuum. $E_{k}$ and $E_{l}$ are the pump and Brillouin Stokes fields with angular frequencies $\omega_{1,2}$ and axial wavevectors $k_{1,2}$. The radiation pressure has been neglected given the size of PCF core. We also assume in our model a counter-propagating wavevector interaction that stands for SBS. We also consider the phonon lifetime by taking into account the elastic losses assuming a complex tensor. We then applied the Green function method and used the Galerkin nodal finite element method (FEM) to transform the integral Eq. 1 into a linear matrix system (for details, See Ref. [15]). Solutions of this system yield rigorous distribution of axial and transverse displacements induced by electrosctriction within the fiber. We finally plotted the associated kinetic energy density as a function of frequency detuning which can directly be compared to experimental Brillouin spectra. Figures 4(a)-(d) shows numerical Brillouin spectra for four decreasing core diameters of the tapered $\mathrm{PCF}$ and also for the fast and slow polarization axes. First, one can see a strong leftward shift of the Brillouin spectrum around

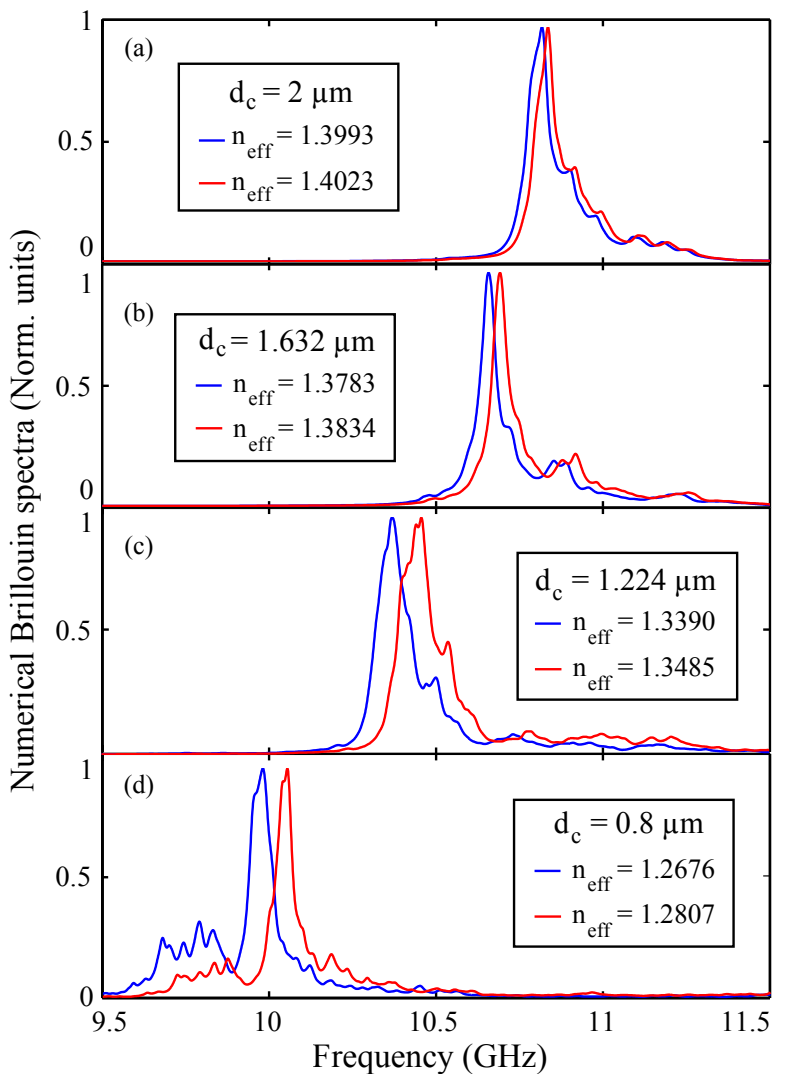

Fig. 4. Numerical simulations of the Brillouin spectrum of the tapered PCF for four increasing core diameters from 0.8 $\mu \mathrm{m}$ to $2 \mu \mathrm{m}$, and for the slow (blue) and fast (red) polarization axis. The insets denote the core size and the effective index.

$1 \mathrm{GHz}$ due to the taper. This range is in good agreement with the above analytical formula of the BFS variation. Second, we can also see the birefringence-induced BFS shift which increases as the core size decreases. More importantly, the Brillouin spectrum broadens and becomes multimode as the fiber core decreases. This can be seen in Fig. 4(d) for a small core diameter of $0.8 \mu \mathrm{m}$ below the optical wavelength. The SBS spectrum features several resonance peaks spanning over about $500 \mathrm{MHz}$. Clearly, this is due to the generation of hybrid acoustic modes, as reported in Ref $[5,8]$. For completeness, we plotted in Fig. 5 the fundamental optical modes and the kinetic energy density of the small and large core of the PCF. We can see that the small optical mode overlaps and interacts with the air-hole microstructure whereas other does not. The sound density is also completely different for the two cases.

Finally, we have measured and compared the SBS threshold power $\mathrm{P}_{\text {th }}$ between the tapered and untapered $\mathrm{PCF}$, using the method described in Ref. [16]. Figure 6 shows the backscattered signal power versus the input pump power for both fibers. We found from these power diagrams a threshold of $28.9 \mathrm{dBm}$ for the $124 \mathrm{~m}$-long tapered fiber, whereas it was only $26 \mathrm{dBm}$ for the $47 \mathrm{~m}$-long untapered fiber. This comparison confirms the high SBS 

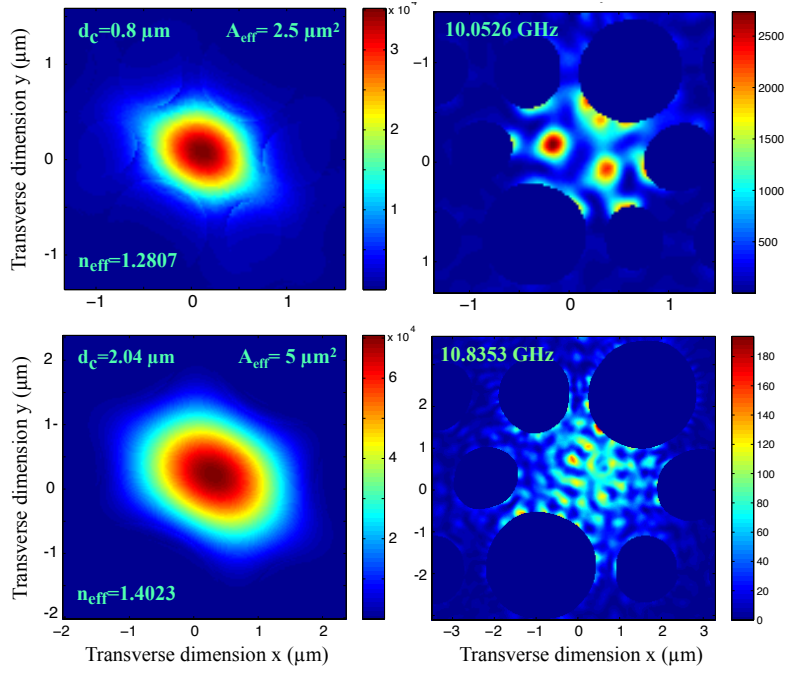

Fig. 5. Numerical simulations of the fundamental optical modes and the kinetic energy density for the two small and large PCF core diameters, $0.8 \mu \mathrm{m}$ and $2 \mu \mathrm{m}$, respectively

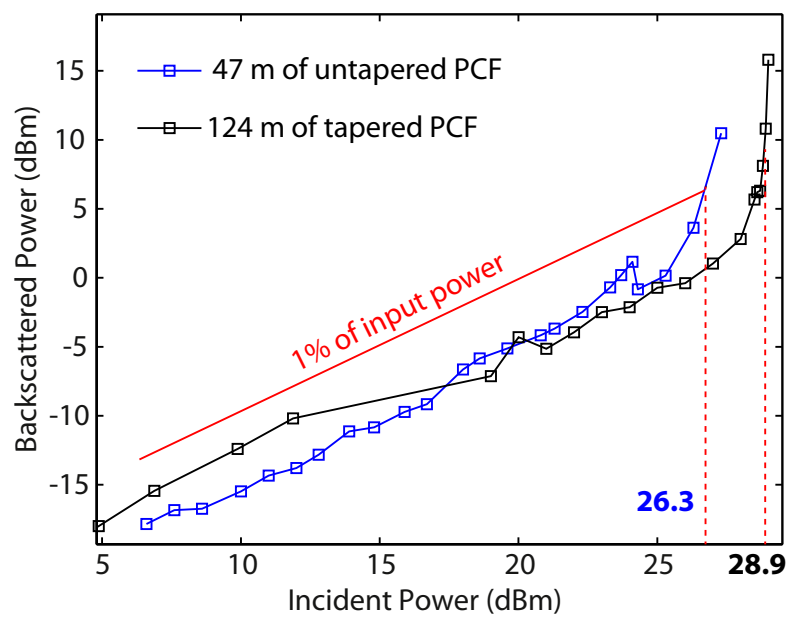

Fig. 6. Backscattered optical power versus the incident pump power for both the untapered (blue) fiber and tapered (black) fiber. The threshold is measured at $1 \%$ of the pump power.

threshold due to the fiber tapering.

In conclusion, we have investigated the stimulated Brillouin scattering in a core-decreasing silica photonic crystal fiber at $1.55 \mu \mathrm{m}$ and we have observed a strong increase of the SBS threshold associated with a broad and multimode Brillouin gain spectrum. We have numerically identified this multi-peaked dynamics as the result of fiber tapering. This type of microstructured fiber with varying core geometry thus opens another way for controlling and shaping the Brillouin gain spectrum and it can find potential applications for mitigating SBS in fiber laser or amplifiers.

This work was supported by the OASIS project (ANR-14-CE36-0005-01), the Région de Franche-Comté, and the LABEX ACTION program (ANR-11-LABX0001-01). A.K. acknowledges support from the TOP-
WAVE (ANR-13-JS04-0004) project, from the "Fonds Européen de Développement Economique Régional", the Labex CEMPI (ANR-11-LABX-0007) and Equipex FLUX (ANR-11-EQPX-0017) through the "Programme Investissements d'Avenir".

\section{References}

1. A. Kobyakov, M. Sauer, and D. Chowdhury, Adv. Opt. Photon. 2, 1 (2010).

2. S. Chin, L. Thevenaz, J. Sancho, S. Sales, J. Capmany, P. Berger, J. Bourderionnet, and D. Dolfi, Opt. Exp. 18, 22599 (2010).

3. D. Marpaung, B. Morrison, M. Pagani, R. Pant, D.-Y. Choi, B. Luther-Davies, S. J. Madden, and B. J. Eggleton, Optica 2, 76 (2015).

4. C. A. Galindez-Jamioy and J. M. Lopez-Higuera, Journal of Sensors 2012, ID 204121 (2012).

5. P. Dainese, P. St. J. Russell, N. Joly, J. C. Knight, G. S. Wiederhecker, H. L. Frangnito, V. Laude and A. Khelif, Nature Physics 2, 388 (2006).

6. J. C. Beugnot, T. Sylvestre, D. Alasia, H. Maillotte, V. Laude, A. Monteville, L. Provino, N. Traynor, S. F. Mafang, and L. Thévenaz, Opt. Exp. 15, 15517 (2007).

7. Benjamin J. Eggleton, Christopher G. Poulton, and Ravi Pant, Adv. Opt. Photon. 5, 536 (2013).

8. Peter T. Rakich, Charles Reinke, Ryan Camacho, Paul Davids, and Zheng Wang, Phys. Rev. X 2, 011008 (2012).

9. R Van Laer, B Kuyken, D Van Thourhout, R Baets, Nat. Phot. 9 (3), 199 (2015).

10. J.-C. Beugnot, S. Lebrun, G. Pauliat, H. Maillotte, V. Laude, T. Sylvestre, Nat. Commun. 5, 5242 (2014).

11. R. I. Woodward, E. J. R. Kelleher, S. V. Popov, and J. R. Taylor, "Stimulated Brillouin scattering of visible light in small-core photonic crystal fibers," Opt. Lett. 39, 2330 (2014).

12. C. Wolff, M. J. Steel, and C. G. Poulton, Opt. Express 22, 32489-32501 (2014).

13. A. Minardo, R. Bernini, W. Urbanczyk, J. Wojcik, N. Gorbatov, M. Tur, and L. Zeni, Opt. Lett. 33, 2329 (2008).

14. J. E. McElhenny, R. Pattnaik, and J. Toulouse, J. Opt. Soc. Am. B 25, 2107 (2008).

15. J.-C. Beugnot and V. Laude, Phys. Rev. B 86, 224304 (2012).

16. B. Stiller, A. Kudlinski, M. W. Lee, M. Delqué, J.-C. Beugnot, G. Bouwmans, H. Maillotte and T. Sylvestre, IEEE Photon. Technol. Lett. 24, pp 667 (2012). 


\section{Complete list of references including titles}

\section{References}

1. Andrey Kobyakov, Michael Sauer, and Dipak Chowdhury, "Stimulated Brillouin scattering in optical fibers," Adv. Opt. Photon. 2, 1-59 (2010).

2. S. Chin, L. Thevenaz, J. Sancho, S. Sales, J. Capmany, P. Berger, J. Bourderionnet, and D. Dolfi, "Broadband true time delay for microwave signal processing, using slow light based on stimulated Brillouin scattering in optical fibers," Opt. Express 18, 22599-22613 (2010).

3. D. Marpaung, B. Morrison, M. Pagani, R. Pant, D.-Y. Choi, B. Luther-Davies, S. J. Madden, and B. J. Eggleton, "Low-power, chip-based stimulated Brillouin scattering microwave photonic filter with ultrahigh selectivity," Optica 2, 76-83 (2015).

4. C. A. Galindez-Jamioy and J. M. Lopez-Higuera, "Brillouin Distributed Fiber Sensors: An Overview and Applications," Journal of Sensors, 2012, ID 204121 (2012).

5. P. Dainese, P. St. J. Russell, N. Joly, J. C. Knight, G. S. Wiederhecker, H. L. Frangnito, V. Laude and A. Khelif, "Stimulated Brillouin scattering from multi-GHz-guided acoustic phonons in nanostructured photonic crystal fibres-guided acoustic phonons in nanostructured photonic crystal fibres," Nature Physics 2, 388-392 (2006).

6. J. C. Beugnot, T. Sylvestre, D. Alasia, H. Maillotte, V. Laude, A. Monteville, L. Provino, N. Traynor, S. F. Mafang, and L. Thévenaz, "Complete experimental characterization of stimulated Brillouin scattering in photonic crystal fiber," Opt. Express 15, 15517-15522 (2007).

7. R. I. Woodward, E. J. R. Kelleher, S. V. Popov, and J. R. Taylor, "Stimulated Brillouin scattering of visible light in small-core photonic crystal fibers," Opt. Lett. 39, 2330-2333 (2014).

8. J.-C. Beugnot, S. Lebrun, G. Pauliat, H. Maillotte, V. Laude, T. Sylvestre, "Brillouin light scattering from surface acoustic waves in a subwavelengthdiameter optical fibre." Nat. Commun. 5, 5242 (2014).

9. Peter T. Rakich, Charles Reinke, Ryan Camacho, Paul Davids, and Zheng Wang, "Giant Enhancement of Stimulated Brillouin Scattering in the Subwavelength Limit," Phys. Rev. X 2, 011008 (2012).

10. R Van Laer, B Kuyken, D Van Thourhout, R Baets, "Interaction between light and highly confined hypersound in a silicon photonic nanowire," Nat. Phot. 9 (3), 199-203 (2015).

11. Benjamin J. Eggleton, Christopher G. Poulton, and Ravi Pant, "Inducing and harnessing stimulated Brillouin scattering in photonic integrated circuits," Adv. Opt. Photon. 5, 536-587 (2013).

12. C. Wolff, M. J. Steel, and C. G. Poulton, "Formal selection rules for Brillouin scattering in integrated waveguides and structured fibers," Opt. Express 22, 32489-32501 (2014).

13. A. Minardo, R. Bernini, W. Urbanczyk, J. Wojcik, N. Gorbatov, M. Tur, and L. Zeni, "Stimulated Brillouin scattering in highly birefringent microstruc- ture fiber: experimental analysis," Opt. Lett. 33, 2329-2331 (2008).

14. John E. McElhenny, Radha Pattnaik, and Jean Toulouse, "Polarization dependence of stimulated Brillouin scattering in small-core photonic crystal fibers," J. Opt. Soc. Am. B 25, 2107-2115 (2008).

15. J.-C. Beugnot and V. Laude, "Electrostriction and guidance of acoustic phonons in optical fibers," Phys. Rev. B 86, 224304 (2012).

16. B. Stiller, A. Kudlinski, M. W. Lee, M. Delqué, J.-C. Beugnot, G. Bouwmans, H. Maillotte and T. Sylvestre, "SBS mitigation in a microstructured optical fiber by varying periodically the core diameter," IEEE Photon. Technol. Lett. Vol. 24, Issue 8, pp 667-669 (2012). 